\title{
Otimização de Sistemas PSK com Codificação Wavelet em Canais com Desvanecimento Plano
}

\author{
Talles R. Ferreira, Sandro G. da Silva, Luiz F. Q. Siveira e Luiz G. de Q. Silveira Júnior
}

\begin{abstract}
Resumo - Desde o seu advento, a codificação wavelet tem sido reconhecida como uma técnica promissora para transmissão digital em sistemas de comunicações sem fio, devido a sua baixa complexidade de decodificação e ao seu bom desempenho sobre canais com desvanecimento plano. Entretanto, o desempenho desta técnica é fortemente influenciado pelo esquema de modulação empregado. Nesse trabalho, é proposto o uso do algoritmo genético e o emprego de ferramentas matemáticas especialmente concebidas na busca por novos esquemas de quantização de símbolos wavelets e na obtenção de novas constelações de sinais PSK, adequadas ao mapeamento sub-ótimo destes símbolos. Os resultados obtidos, através de simulações computacionais, mostram a eficácia do método proposto e indicam que o seu emprego pode levar a novas alternativas para explorar o potencial da codificação wavelet em sistemas de comunicações sujeitos ao desvanecimento.
\end{abstract}

Palavras-Chave-Modulação, Codificação Wavelet, Projeto de Constelações de Sinais, Algoritmos Genéticos.

Abstract - Since its advent, wavelet coding has been recognized as a promising technique for digital transmission over wireless communication environments, especially due to its low decoding complexity and good performance over multipath fading. However, this technique generates not-equiprobable symbols, and consequently the performance gains from these systems are influenced significantly by their modulation schemes. In this work, it is proposed a design methodology to obtain sub-optimum modulation schemes for wavelet systems over Rayleigh fading channels. In this context, novels signal constellations and quantization schemes are obtained via genetic algorithm and mathematical tools. Numerical results obtained from simulations show that the wavelet-coded systems derived here have very good performance characteristics over fading channels.

Keywords - Modulation, Wavelet Coding, Signal Constellation Design, Genetic Algorithm.

\section{INTRODUÇÃO}

Em sistemas de comunicações sem fio, o efeito do multipercurso, chamado de desvanecimento, pode comprometer de forma severa o desempenho destes sistemas. Com o objetivo de minimizar seus efeitos, várias estratégias tem

Talles R. Ferreira e Sandro G. da Silva, Departamento de Engenharia Elétrica, Universidade Federal do Rio Grande do Norte, Natal, Brasil E-mails:tallesrferreira@yahoo.com.br, sgsee@ufrnet.br Luiz F. Q. Silveira, Instituto Federal de Educação, Ciência e Tecnologia do Rio Grande do Norte, Natal, Brasil, E-mail: lfelipe@cefetrn.br, Luiz G. de Q. Silveira Júnior, Departamento de Ciências Exatas e Naturais da Universidade Federal Rural do Semi-Árido, Mossoró-RN, Brasil, E-mail: junior@ufersa.edu.br sido propostas. Neste sentido, é crescente o número de aplicações que utilizam wavelets visando combater os efeitos degenerativos destes canais, tais como novos esquemas de modulação e técnicas de codificação de canal [1], [2], [3], [4].

O primeiro trabalho de codificação de canal com wavelets foi proposto por Tzannes [5] como uma nova alternativa para combater os efeitos do multipercurso. A codificação wavelet se baseia nas propriedades de ortogonalidade entre as linhas de uma matriz de coeficiente wavelets $(\mathrm{MCW})$. Os símbolos produzidos na saída do codificador, denominados de símbolos wavelets, são nãoeqüiprováveis e assumem diversos níveis [6]. No processo de codificação wavelet, cada bit de informação que entra no codificador, é multiplicado, de maneira sucessiva, pelos coeficientes de uma das linhas da MCW, disseminando a informação de cada bit sobre vários símbolos codificados.

Devido às propriedades de ortogonalidade entre as linhas das MCW, a sequência de bits de informação pode ser recuperada de forma simples, usando um banco de correlatores formados a partir das linhas da $\mathrm{MCW}$ utilizada na codificação. O mecanismo de espalhamento da informação no tempo, antes da transmissão e o recolhimento dela no receptor, contribui para melhorar a robustez do sistema de comunicação à combinação de efeitos do desvanecimento plano variante no tempo e de ruído localizado.

Uma das vantagens proporcionadas pelo emprego da codificação wavelet é a possibilidade que esta oferece de aumentar o ganho de diversidade temporal, melhorando o desempenho do sistema de comunicações frente aos efeitos destrutivos do canal com desvanecimento Rayleigh. A obtenção de melhores ganhos de diversidade é proporcionada pelo emprego de uma matriz wavelet de maiores dimensões, que implica no espalhamento da informação de cada bit sobre um número maior de símbolos wavelets. Desta forma, o desempenho do sistema é influenciado pelo número de colunas da MCW utilizada.

Neste contexto, o emprego de matrizes wavelets de maiores dimensões pode ocasionar um acúmulo de pontos na constelação de sinais utilizada no esquema de modulação, podendo comprometer severamente o desempenho do sistema.

Assim, para viabilizar a obtenção de melhores ganhos de diversidade por parte dos sistemas que empregam a codificação wavelet, é fundamental, durante o projeto do esquema de modulação, dispor de ferramentas e métodos eficientes na busca por bons esquemas de mapeamento. Em [7], foi proposto o uso de um algoritmo genético (AG) 
na busca de constelações de sinais PSK adequadas ao mapeamento de símbolos wavelets, obtidos a partir de um esquema de quantização previamente fixado. Esta busca é baseada em uma análise feita anteriormente sobre a probabilidade de erro do decodificador [8].

De fato, o projeto de constelações é um problema típico de otimização combinatória que consiste em minimizar uma função custo que modela o desempenho do sistema codificado, e que tem por argumento, os parâmetros da constelação de sinais. Os algoritmos genéticos, quando aplicados na solução de problemas desse tipo, podem encontrar soluções de alta qualidade, ainda que sem garantia de solução ótima, em um tempo muito reduzido quando comparado ao tempo necessário para avaliar todas as soluções possíveis [7].

Este artigo discute o uso do algoritmo genético proposto em [7], na avaliação dos esquemas de quantização de símbolos wavelets, os quais passam a ser resultado da sobreposição de sinais observada na constelação obtida via AG. Este esquema de quantização então obtido, explora o fato de que num sistema de comunicações baseado na codificação wavelet, mesmo que certos símbolos nunca sejam transmitidos, isto não implica necessariamente numa degradação significativa no desempenho do receptor (demodulador e decodificador wavelet).

Especificamente, este artigo trata do projeto de esquemas de quantização de símbolos wavelets. Os sistemas wavelets aqui avaliados são baseados nas MCWs $2 \times 32$ e $2 \times 128$. Além disso, também serão apresentadas as constelações de sinais obtidas a partir destes esquemas de quantização.

A eficácia do método proposto será avaliada em termos do desempenho de cada sistema sobre um canal sujeito ao desvanecimento Rayleigh plano, via simulação computacional. Conforme será mostrado, os resultados obtidos comprovam que a técnica aqui desenvolvida leva a obtenção de um esquema de quantização eficaz para matrizes de coeficientes wavelets de dimensões elevadas.

O restante deste artigo está organizado como segue. A Seção II apresenta uma revisão da técnica de codificação wavelet. Na Seção III é apresentado o modelo do sistema que será objeto de otimização. A seção IV é dedicada a apresentar os detalhes do projeto dos esquemas de quantização dos símbolos wavelets. Os resultados numéricos obtidos com a simulação do esquema proposto são mostrados e discutidos na Seção V. Finalmente, a Seção VI apresenta as conclusões deste trabalho.

\section{Fundamentos da CodificaÇÃo Wavelet}

A codificação wavelet utiliza as linhas da matriz de coeficientes wavelets (MCW) com o objetivo de codificar os bits de informação. Como mostrado em [6], uma MCW de ordem $m$ e gênero $g$ tem dimensão $m \times m g$, denotada por

$$
\mathbf{A}=\left(\begin{array}{ccc}
a_{0}^{0}, & \ldots, & a_{m g-1}^{0} \\
a_{0}^{1}, & \ldots, & a_{m g-1}^{1} \\
\vdots & & \vdots \\
a_{0}^{m-1}, & \ldots, & a_{m g-1}^{m-1}
\end{array}\right)
$$

cujas as entradas podem pertencer ao conjunto dos números inteiros, reais, ou complexos.

Em particular, uma matriz MCW é denominada real e plana, quando seus coeficientes são números reais escolhidos no conjunto $\{1,-1\}$, e satisfazem as seguintes condições $[6]$ :

$$
\begin{gathered}
\sum_{k=0}^{m g-1} a_{k}^{j}=m \sqrt{g} \delta_{0, j}, \quad 0 \leq j \leq m-1 \\
\sum_{k} a_{k+m l}^{j} a_{k+m l^{\prime}}^{j^{\prime}}=m g \delta_{j, j^{\prime}} \delta_{l, l^{\prime}}, \\
0 \leq j, j^{\prime} \leq m-1 \\
0 \leq l, l^{\prime} \leq g-1
\end{gathered}
$$

sendo $\delta_{j, j^{\prime}}$ o delta de Kronecker.

A propriedade fundamental de MCWs para a codificação de canal é dada pela Equação 3. Esta equação afirma que as linhas de uma MCW de ordem $m$ são mutuamente ortogonais sobre deslocamentos de comprimento $\mathrm{lm}$, sendo $0 \leq l \leq g-1$. Esta também afirma que cada linha é ortogonal a uma cópia de si mesma, quando deslocada por $l m$, sendo $0<l \leq g-1$. Na teoria de bancos de filtros digitais, as linhas $\left\{a^{j}\right\}$ representam filtros passa-faixa de um banco de filtros com $m$-bandas.

O símbolo wavelet gerado no intevalo de tempo $n=$ $p m+q$, é dado por:

$$
y_{p m+q}=\sum_{j=0}^{m-1} \sum_{l=0}^{g-1} a_{l m+q}^{j} x_{(p-l) m+j},
$$

e assume valores no alfabeto de símbolos wavelets definidos no conjunto $y=\{-m g,-m g+$ $2, \ldots,-2 k, \ldots, 0,2 k, \ldots, m g\}$, com cardinalidade igual a $m g+1$. Consequentemente, os símbolos wavelets são multiníveis e dependem de diversos bits de informação.

\section{Modelo do Sistema}

A Figura 1 ilustra o modelo do sistema com codificação wavelet adotado neste trabalho. Neste sistema, a fonte de informação gera uma sequência de bits de informação $x_{n}$ estatísticamente independentes e equiprováveis. Essa seqüência de bits é então codificada por uma MCW real e plana de dimensões $m \times m g$.

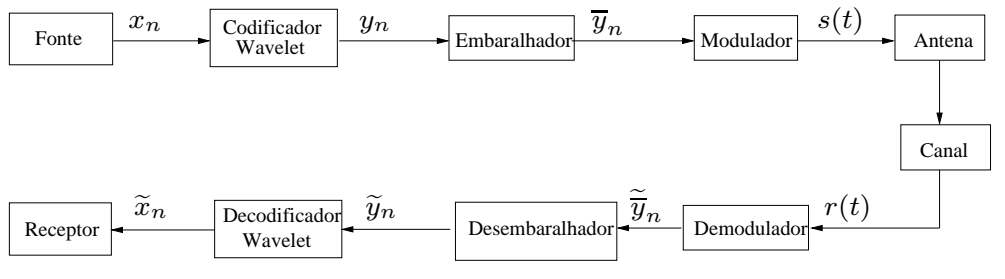

Fig. 1. Modelo do sistema de comunicações baseado na codificação com matrizes wavelets

Após o embaralhamento, os símbolos wavelets $\bar{y}_{n}$ são modulados utilizando uma constelação PSK de raio unitário com $m g+1$ pontos, sendo cada símbolo wavelet mapeado em um ponto da constelação, e transmitidos por 
uma única antena sobre um canal com desvanecimento Rayleigh. O canal é assumido ser plano e essencialmente constante durante um intervalo de sinalização, apesar de ser variante no tempo. É importante observar que $n$ bits de informação são codificados em $n$ símbolos wavelets e enviados durante $n$ intervalos de sinalização, de tal forma que a eficiência espectral de $1 \mathrm{bit} / \mathrm{seg} / \mathrm{Hz}$ é facilmente assegurada no modelo do sistema de comunicações avaliado neste trabalho.

A saída do canal em tempo discreto é modelada pela variável aleatória complexa $r_{n}=\alpha_{n} s_{n}+w_{n}$, sendo $\alpha_{n}$ a amostra de desvanecimento, $s_{n}$ o símbolo transmitido, e $w_{n}$ o ruído complexo gaussiano branco, de média nula e partes real e imaginária de mesma variância $N_{0} / 2$.

Na recepção, considera-se que o receptor tem perfeita informação sobre o estado do canal, ou seja, ele conhece o valor exato do desvanecimento Rayleigh em cada intervalo de sinalização.

A regra de decisão empregada pelo demodulador foi o critério MAP. Após a demodulação, as estimativas dos sinais recebidos são inversamente mapeadas nos símbolos $\widetilde{y}_{n}$. A estimativa $\widetilde{y}_{n}$ é dada por $\widetilde{y}_{n}=y_{n}+e_{n}$, em que $e_{n}$ é uma variável aleatória discreta, denominada ruído de demodulação [8].

Em [8], o sistema com codificação wavelet considerado aqui foi analisado, em termos de sua probabilidade de erro de bit (BER), através do uso de funções geradoras de momentos. Especificamente, a probabilidade de erro de bit desse sistema foi analisada a partir do ruído de demodulação, $e_{n}$, que na recepção é decodificado pelo decodificador wavelet, junto com o símbolo wavelet efetivamente transmitido $y_{n}$. Neste contexto, a probabilidade de erro de bit do sistema com codificação wavelet, pode ser expressa por:

$$
\begin{array}{r}
P_{e}=\sum_{k=1}^{\frac{m g(2 m g-1)}{2}} \operatorname{Pr}\left(v_{i}=m g+2 k \mid x_{i-(m g-1)}=-1\right) \\
+\quad 0.5 \operatorname{Pr}\left(v_{i}=m g \mid x_{i-(m g-1)}=-1\right),
\end{array}
$$

em que $v_{i}$ é uma variável aleatória discreta, denominada ruído wavelet, que modela a decodificação wavelet da parcela de distorção introduzida pelo canal (ruído de demodulação), $m$ é o posto e $g$ é o gênero da MCW utilizada no processo de codificação.

\section{Projeto de Esquemas de Quantização}

Nesta seção, é apresentado o projeto de um esquema de quantização para sistemas com codificação wavelet, que utilizam as MCW $2 \times 32$ e MCW $2 \times 128$, como matrizes de codificação.

Em [7] foram projetadas constelações de sinais para o uso nos sistemas com codificação wavelet, por meio de um algoritmo genético com o objetivo de minimizar a probabilidade de erro do sistema. Após o projeto destas constelações, a melhor constelação encontrada pelo AG, em alguns casos, tinha alguns dos seus sinais sobrepostos.
Percebeu-se que a sobreposição dos sinais na constelação sugeriria uma quantização natural dos símbolos wavelets. Estes resultados indicavam que o algoritmo genético poderia ser utilizado para o projeto dos esquemas de quantização de símbolos wavelets para sistemas com MCW de grandes dimensões.

Então, ao invés de se utilizar um método de tentativa e erro empírico, baseado em simulações de Monte Carlo, para se projetar os esquemas de quantização e só a partir deste, a constelação de sinais, poder-se-ia aplicar o AG para se projetar uma constelação com todos os mg +1 símbolos passíveis de serem gerados pela codificação, sem nenhum esquema prévio de quantização. Como o algoritmo genético sempre irá buscar a melhor constelação para uma dada situação, naturalmente a melhor constelação encontrada pelo AG já teria uma configuração com sinais sobrepostos, que poderia então ser utilizada para se definir o esquema de quantização dos símbolos wavelets. Essa é a idéia chave que está por traz da metodologia de projeto desenvolvida nesse trabalho.

Neste trabalho, será apresentado uma metodologia de projeto de esquemas de modulação PSK para uso em conjunto com a codificação wavelet. Os parâmetros de projeto são os ângulos dos sinais da constelação PSK, com a restrição da energia média da constelação ser unitária. O objetivo é determinar qual é o conjunto de parâmetros que minimiza a probabilidade de erro de bit do sistema wavelet.

O algoritmo genético desenvolvido em [9], e adotado neste trabalho, permite o uso de dois tipos de representação: binária e real. No caso do projeto de constelações de sinais, os genes dos cromossomos são os ângulos dos sinais da constelação PSK, representados em radianos. Para este caso, a representação continua é a mais indicada, sendo esta, a representação escolhida na configuração do algoritmo. De acordo com essa representação, cada cromossomo (indivíduo da população) é representado por um vetor $x=\left(x_{1}, x_{2}, x_{3}, \ldots, x_{k}\right) \in \Re^{k}$.

A população inicial é criada de forma aleatória e o número de cromossomos (indivíduos) da população inicial é mantido nas populações seguintes. Os genes de cada cromossomo são selecionados a partir de uma distribuição uniforme $U\left(a_{i}, b_{i}\right)$ em que $a_{i}$ e $b_{i}$ são respectivamente os limites inferiores e superiores do espaço de busca, para os valores de cada gene $x_{i}$ do cromossomo $\mathbf{x}$. Note que para uma população de cromossomos $k$-dimensionais, deve-se fornecer ao algoritmo uma matriz com extremos I dada por (6), a qual irá definir o espaço de soluções válidas para o problema.

$$
I=\left[\begin{array}{cc}
a_{1} & b_{1} \\
a_{2} & b_{2} \\
\vdots & \vdots \\
a_{k} & b_{k}
\end{array}\right]
$$

Os operadores genéticos utilizados aqui foram: elitismo, seleção geométrica normalizada, cruzamento heurístico e mutação não uniforme multi-dimensional [9]. O critério de 
parada adotado neste trabalho para o algoritmo genético foi o número máximo de gerações. O número máximo de gerações variou entre 500 a 1000 gerações.

No projeto de constelações para sistemas de transmissão, a função objetivo é definida em termos da probabilidade de erro do sistema, e a meta do método de otimização é a minimização desta função objetivo. Em se tratando do projeto das constelações para sistemas de transmissão baseados na codificação com matrizes wavelets, a função objetivo a ser minimizada é dada pela Equação (5).

Com base na metodologia proposta, o primeiro passo foi aplicar o AG para projetar uma constelação para o sistema com MCW de dimensões $2 \times 32$, com a finalidade de se encontrar uma constelação de sinais sobrepostos e a partir desta, definir um esquema de quantização para os símbolos wavelets gerados por essa MCW na codificação.

A melhor constelação encontrada pelo AG, depois de varias simulações, é apresentada na Figura 2. Esta constelação foi otimizada para uma relação sinal ruído (SNR) de 15 dB. A Figura 2 ilustra a constelação encontrada pelo AG para o sistema com MCW $2 \times 32$. Deve ser observado que a constelação obtida apresenta uma nítida sobreposição entre os seus 33 pontos, cujos ângulos são apresentados na Tabela I.

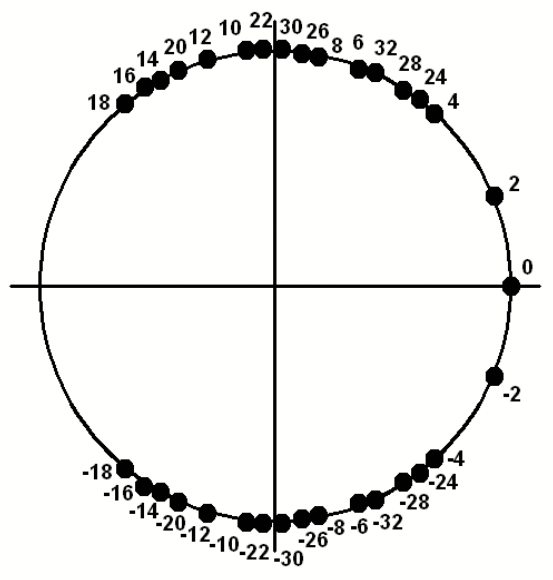

Fig. 2. Constelação 33-PSK para uma MCW $2 \times 32$

Em seguida, na obtenção do esquema de quantização associado, foi feita uma nova simulação do sistema com MCW $2 \times 32$, onde todos os 33 símbolos que podiam ser gerados pela MCW $2 \times 32$ foram mapeados pelos sinais da constelação 33-PSK da Figura 3, e transmitidos sobre um canal sem ruído. A demodulação foi realizada pelo critério MAP com parâmetro $N_{0}=10^{-1,5}$. Deve ser observado que esse valor de $N_{0}$ corresponde a uma relação sinal-ruído de transmissão igual a $15 \mathrm{~dB}$.

Devido à distorção nos limiares das regiões de decisão causada pelo critério MAP com $N_{0}=10^{-1,5}$, alguns dos símbolos transmitidos foram sempre demodulados pelos seus símbolos vizinhos com maiores probabilidades de ocorrência. A troca de símbolos durante uma demodulação sem ruído de transmissão indica uma quantização natural
TABELA I

Ângulos dos sinais da CONStelaÇÃo 33 - PSK.

\begin{tabular}{|c|c|}
\hline Sinais da Constelação & Ângulos \\
\hline \hline 2 & $23^{\circ}$ \\
4 & $48^{\circ}$ \\
6 & $66^{\circ}$ \\
8 & $83^{\circ}$ \\
10 & $96^{\circ}$ \\
12 & $107^{\circ}$ \\
14 & $115^{\circ}$ \\
16 & $119^{\circ}$ \\
18 & $130^{\circ}$ \\
20 & $114^{\circ}$ \\
22 & $93^{\circ}$ \\
24 & $52^{\circ}$ \\
26 & $85^{\circ}$ \\
28 & $56^{\circ}$ \\
30 & $89^{\circ}$ \\
32 & $65^{\circ}$ \\
\hline
\end{tabular}

dos símbolos wavelets, ocasionada pela regra de demodulação MAP. Foi a partir dessa observação que o esquema de quantização apresentado na Tabela II foi definido.

TABELA II

QUANTIZAÇÃO PARA MCW $2 \times 32$.

\begin{tabular}{|c|c|}
\hline Conjunto & Símbolos \\
Truncado & Representativos \\
\hline \hline$\{0\}$ & 0 \\
$\{2\}$ & 2 \\
$\{-2\}$ & -2 \\
$\{4,24,28\}$ & 4 \\
$\{-4,-24,-28\}$ & -4 \\
$\{6,32\}$ & 6 \\
$\{-6,-32\}$ & -6 \\
$\{8,30\}$ & 8 \\
$\{-8,-30\}$ & -8 \\
$\{10\}$ & 10 \\
$\{-10\}$ & -10 \\
$\{12,20\}$ & 12 \\
$\{-12,-20\}$ & -12 \\
$\{14,16,18\}$ & 14 \\
$\{-14,-16,-18\}$ & -14 \\
\hline
\end{tabular}

Pode ser verificado a partir da Tabela II que o esquema de quantização obtido requer uma constelação com 15 sinais. A constelação casada a este esquema de quantização pode ser construída excluindo-se aqueles sinais que mapeiam os símbolos truncados, já que estes sinais nunca serão demodulados, e mantendo-se os sinais que mapeiam os símbolos representativos. A Figura 3 apresenta a constelação 15-PSK obtida utilizando-se esse procedimento.

Seguindo-se os mesmos passos anteriormente definidos, utilizou-se um AG para se projetar uma constelação 129-PSK de sinais sobrepostos para o sistema com MCW $2 \times 128$, e a partir desta, definir os esquemas de quantização apresentados na Tabela III e a constelação 29-PSK apresentada na Figura 4.

\section{Resultados}

Nesta seção são apresentados os resultados de desempenho obtidos através da simulação computacional dos sistemas com codificação wavelet com MCW $2 \times 32 \mathrm{MCW}$ 


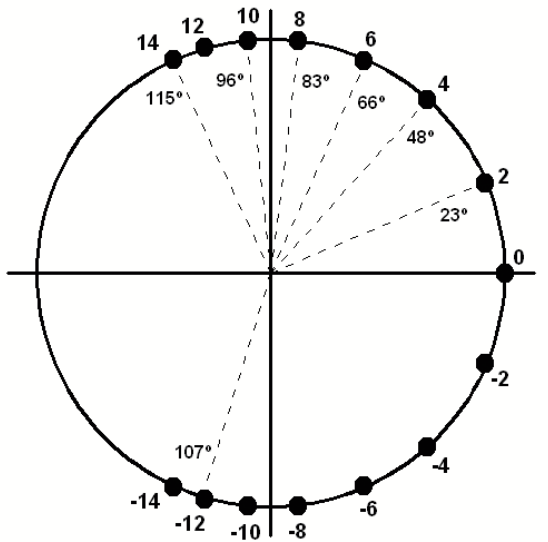

Fig. 3. Constelação 15-PSK para uma MCW $2 \times 32$.

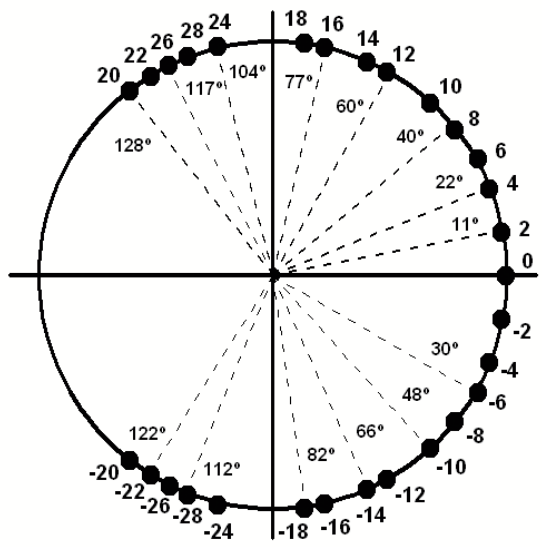

Fig. 4. Constelação 29-PSK para uma MCW $2 \times 128$.

$2 \times 128$ empregando os esquemas de modulação obtidos na seção IV. O sistema foi simulado em canais com desvanecimento Rayleigh perfeitamente entrelaçados e perfeitamente estimados na recepção.

A Figura 5 apresenta as curvas de desempenho do sistema com MCW $2 \times 32$ utilizando-se os esquemas de modulação 33-PSK e 15-PSK. Como referência, se encontra também na Figura 5 a curva obtida de [8] para o seu sistema wavelet com modulação PSK e MCW $2 \times 32$. A partir dos resultados obtidos, observa-se que o esquema de modulação 33-PSK projetado via AG obteve um ganho de desempenho em relação ao sistema de referência. Além disso, pode ser visto ainda na Figura 5, que o esquema de modulação 15-PSK obteve um resultado similar ao esquema 33-PSK, mesmo utilizando um esquema de quantização para os símbolos wavelets. Isto mostra que o algoritmo genético é um método eficaz para se definir esquemas de quantização para os símbolos wavelets.

A Figura 6 apresenta as curvas de desempenho do sistema com MCW $2 \times 128$ utilizando os esquemas de modulação 129-PSK e 29-PSK. Como referência, se encontra também na Figura 6 o desempenho obtido por [7] para
TABELA III

QUANTIZAÇÃO PARA MCW $2 \times 128$.

\begin{tabular}{|c|c|}
\hline $\begin{array}{l}\text { Conjunto } \\
\text { Truncado }\end{array}$ & $\begin{array}{c}\text { Símbolos } \\
\text { Representativos }\end{array}$ \\
\hline $\begin{array}{c}\{0\} \\
\{2\} \\
\{-2\} \\
\{4,90\} \\
\{-4,-90\} \\
\{6,82,118\} \\
\{-6,-82,-118\} \\
\{8\} \\
\{-8\} \\
\{10,86\} \\
\{-10,-86\} \\
\{12,76,106,110,126\} \\
\{14\} \\
\{-14\} \\
\{12,-76,-106,-110,-126\} \\
\{-16,-46,-48,48,54,60,68,96\} \\
\{18,38,52,66,70,72,80, \\
\ldots 100,116,122,128\} \\
\{-18,-38,-52,-66,-70,-72,-80, \\
\ldots-100,-116,-122,-128\} \\
\{20,44,56,102,114,120\} \\
\{-20,-44,-56,-102,-114,-120\} \\
\{22\} \\
\{-22\} \\
\{24,36,40,50,58,64,74,78, \\
\ldots 88,92,94,98,104,108,112\} \\
\{-24,-36,-40,-50,-58,-64,-74,-78, \\
\ldots-88,-92,-94,-98,-104,-108,-112\} \\
\{26,32,34,62,84,124\} \\
\{-26,-32,-34,-62,-84,-124\} \\
\{28,30,42\} \\
\{-28,-30,-42\} \\
\end{array}$ & $\begin{array}{c}0 \\
2 \\
-2 \\
4 \\
-4 \\
6 \\
-6 \\
8 \\
-8 \\
10 \\
-10 \\
12 \\
-12 \\
14 \\
-14 \\
16 \\
-16 \\
18 \\
\\
-18 \\
20 \\
-20 \\
22 \\
-22 \\
\\
24 \\
\\
-24 \\
26 \\
-26 \\
28 \\
-28 \\
\end{array}$ \\
\hline
\end{tabular}

o sistema com MCW $2 \times 128$ utilizando um esquema de modulação 11-PSK.

A partir da Figura 6, pode-se verificar que o sistema de modulação projetado via $\mathrm{AG}$ tem um desempenho similar aos sistemas de referência para valores de SNR inferiores a $15 \mathrm{~dB}$. Mas a partir desse ponto, à medida que a relação sinal-ruído aumenta, o desempenho do sistema projetado neste trabalho, com 129 sinais na constelação, supera o desempenho do sistema de referência. Com o aumento gradual da relação sinal-ruído, a distorção dos limiares das regiões de decisão diminuem, com isso, alguns símbolos que eram suprimidos pelo critério MAP, passam a ser demodulados corretamente, exigindo uma constelação com mais sinais para não haver perda de informação. E é o que acontece com o sistema de referência, já que utiliza uma constelação com apenas 11 sinais.

Na Figura 6, podemos ainda constatar que o esquema de modulação 29-PSK teve um desempenho similar ao esquema 129-PSK. Isto já era esperado, visto que o sistema baseado numa MCW $2 \times 32$ utilizando o esquema de quantização projetado via $\mathrm{AG}$ também obteve um resultado similar ao mesmo sistema com todos os sinais. Este resultado indica que o AG é capaz de encontrar um esquema de quantização eficaz para matrizes de coeficientes wavelets com dimensões elevadas. 


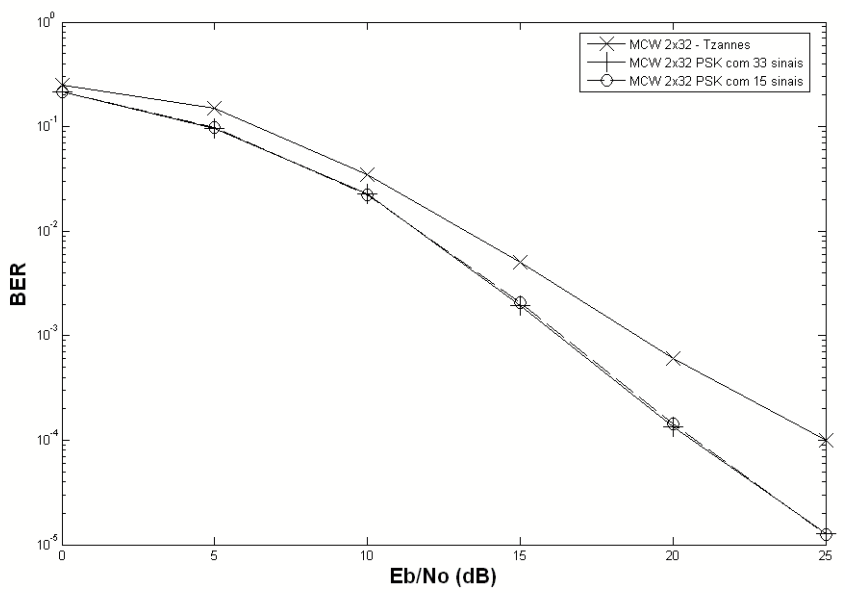

Fig. 5. Desempenho do sistema com MCW $2 \times 32$ e esquemas de modulação PSK com 15 e 33 sinais projetados via AG.

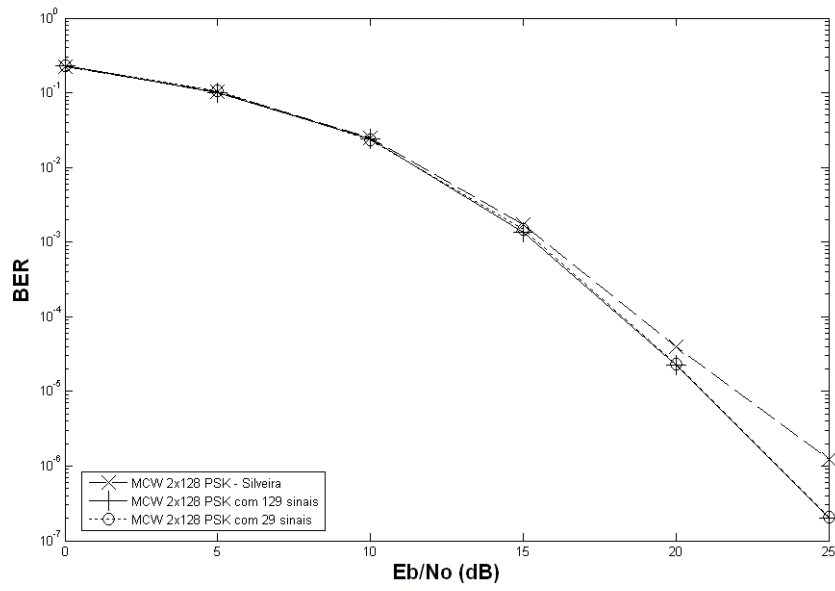

Fig. 6. Desempenho do sistema com MCW $2 \times 128$ com esquemas de modulação PSK com 29 e 129 sinais projetados via AG.

\section{Conclusões}

Neste trabalho foi desenvolvida uma metodologia de projeto e avaliação de esquemas de modulação PSK para sistemas com codificação wavelet submetidos a canais com desvanecimento Rayleigh plano. Especificamente, os esquemas de modulação projetados aqui foram obtidos por um Algoritmo Genético guiado por ferramentas de análise matemática.

Os esquemas de modulação projetados aqui, para sistemas com codificação wavelet com MCWs $2 \times 32$ e $2 \times 128$, obtiveram desempenhos superiores àqueles apresentados em [5] e [7]. Esses resultados evidenciam a eficiência do algoritmo genético tanto para o projeto de constelações quanto para o projeto de esquemas de quantização, independente da dimensão da matriz empregada para o esquema de codificação do sistema.

Tendo em vista os resultados obtidos, a principal contribuição desse trabalho foi propor e consolidar um método eficaz de obtenção de esquemas de quantização, e de constelações de sinais para compor os esquemas de modulação de sistemas que utilizam a codificação wavelet.

\section{REFERÊNCIAS}

[1] G. W. Wornell, "Emerging applications of multirate signal processing and wavelets in digital communications," Proceedings of the IEEE, v. 84, pp. 586-603, April 1996.

[2] F. Daneshgaran, M. Mondin e F. Dovis, "Shaping the power spectrum of TCM codes using wavelet packet modulation," Electronics Letters, v. 35, pp. 1459-1461, August 1999.

[3] V. Mittal, Y. Gautam, R. K. Mallik e S. D. Joshi, "Analysis of Wavelet Modulation in Frequency-Selective Fading," IEEE Trans. on Vehicular Technology , pp. 3818-3826, November 2007.

[4] F. Fekri, S. W. Mclaughlin, R. M. Mersereau e R. W. Schafer, "Block Error Correcting Codes Using Finite-Field Wavelet Transforms," IEEE Trans. on Signal Processing, pp. 991-1004, March 2006.

[5] M. A. Tzannes e M. C. Tzannes, "Bit-by-Bit Channel Coding using Wavelts," Proceedings of IEEE Global Telecommunications Conference GLOBECOM'92, pp. 684-688, 1992.

[6] , H. L. Resnikoff and R. O. Wells Jr., "Wavelet Analysis: The Scalable Structure of Information," Springer-Verlang, New York, 1998.

[7] L. F. Q. Silveira, L. G. de Q Silveira Júnior, F. M. Assis e E. L. Pinto, "Algoritmo Genético Aplicado na Obtenção de Constelações de Sinais para Transmissão Digital com Codificação Wavelet em Canais com Desvanecimento Rayleigh," VIII Congresso Brasileiro de Redes Neurais - CBRN'2007, 2007.

[8] L. F. Q. Silveira, F. M. Assis e E. L. Pinto, "Análise de Desempenho de Sistemas com Codificação Wavelet e Diversidade em Canais com Desvancimento Plano," XXII Simpósio Brasileiro de Telecomunicações - SBrT'2005, 2005.

[9] C. Houck, J. A. Joines e M. G. Kay, "Genetic Algorithm for Function Opitmization: A Matlab Implementation,"http://www.ie.ncsu.edu/mirage/GAToolBox/, 1995. 